\title{
Log-moment estimators of the Nakagami-lognormal distribution
}

\author{
Juan Reig ${ }^{1 *}$ (D), Conor Brennan², Vicent M. Rodrigo Peñarrocha' and Lorenzo Rubio
}

\begin{abstract}
In this paper, estimators of the Nakagami-lognormal (NL) distribution based on the method of log-moments have been derived and thoroughly analyzed. Unlike maximum likelihood (ML) estimators, the log-moment estimators of the NL distribution are obtained using straightforward equations with a unique solution. Also, their performance has been evaluated using the sample mean, confidence regions and normalized mean square error (NMSE). The NL distribution has been extensively used to model composite small-scale fading and shadowing in wireless communication channels. This distribution is of interest in scenarios where the small-scale fading and the shadowing processes cannot be easily separated such as the vehicular environment.
\end{abstract}

Keywords: Wireless, Propagation, Fading, Shadowing, Estimators, Nakagami, Lognormal

\section{Introduction}

In wireless communications, the composite Nakagamilognormal (NL) distribution has been extensively employed to model the mixture of small-scale fading and shadowing [1-14]. This distribution was initially proposed in [1] to obtain the outage probability in scenarios involving multiple co-channel interferers. In several other works, different performance parameters in a NL fading channel using different diversity techniques have been obtained [2-4]. Outage probabilities in diversity channels using non-coherent frequency-shift keying (NCFSK) and differential phase-shift keying (DPSK) matched filter receivers over NL channels were analyzed in [2]. In [3], both the average level crossing rate (LCR) and average time fade duration (AFD) were derived for selection diversity receivers, over an arbitrary number of independent but non-identical NL fading signals. An approximation of both the probability density function (PDF) of the signal-to-noise ratio (SNR) and the average bit error rate (BER) in maximum ratio combining (MRC) receivers over correlated NL channels has been derived by using the two-point lossless moment generating function (MGF) in [4]. The performance of adaptive modulation

\footnotetext{
${ }^{*}$ Correspondence: jreigp@dcom.upv.es

'Electromagnetic Radiation Group (GRE), Institute of Telecommunications and Multimedia Applications (iTEAM), Universitat Politècnica de València, Camino de Vera, 46022 Valencia, Spain

Full list of author information is available at the end of the article
}

systems under MRC over NL channels was analyzed in $[5,6]$. In the last few years, the NL distribution has been employed to model the wireless channel in emerging cellular networks [7-10]. In [7], the performance of relay deployments has been investigated where the NL distribution is used to model the relay link, whereas the access link between a mobile terminal and its serving relay node is modeled by a Rician-lognormal distribution. An adaptive selection/maximal-ratio (ASM) combining system over independent non-identical composite NL fading channels using the mixture gamma distribution has been analyzed in [8] corresponding to cooperative relaying networks. In [9], the authors assess the performance of offload macro users to small macro networks under two different composite fading scenarios, NL channel fading and timeshared shadowed/unshadowed channel fading in the framework of heterogeneous networks with stochastic geometry. The coverage probability and average rate of a typical user in a cellular network under NL channels have been analyzed in [10], modeling the base station deployments with stochastic geometry.

Recently, the interest in the NL distribution has increased due to the difficulty in separating the smallscale fading and the shadowing processes in small stationary scenarios such as vehicular environments $[11,12]$. However, the PDF of the NL distribution involves an infinite integral which can be approximated by a weighted mixture of gamma distributions using Gaussian-Hermite 
integration [13]. In [11], the authors use an approximation of the NL PDF based on an expansion using central differences [15] to evaluate the amount of fading, outage probability and the average channel capacity over a combined NL shadowing and non-shadowing fading channel. An expression in the form of a finite series for the average signal error probability (ASEP) for $M$-ary phase shift keying (MPSK) has been derived for tight bounds in [14]. Moreover, in a measurement campaign conducted in a macrocellular urban environment [12], the NL was the best-fit distribution when compared with other distributions proposed to model the composite small-scale fading and shadowing, namely the Rayleigh-lognormal [16], $\alpha-\mu$ [17] or generalized- $K[18]$.

In order to analyze the performance of cellular networks over different conditions (modulations schemes, diversity techniques, etc) in a given scenario where the propagation channel is modeled as a NL distribution, one first needs to estimate the parameters of the NL distribution in real environments from measurement campaigns. The values of the NL parameters strongly influence the outage probability, ASEP, BER, average channel capacity, and other performance parameters of such a scenario. Therefore, efficient and robust estimators of the NL distribution need to be derived in order to obtain the parameters of this distribution from measurement campaigns.

The NL distribution is a tri-parametric distribution and the integral form of its PDF presents an inherent difficulty in estimating the parameters. To the best of the authors' knowledge, no assessment of the NL estimators has been carried out previously. There are different techniques to estimate the parameters of a distribution. The log-moments method has been proposed to estimate the parameters of other distributions used in wireless communications, such as the Rayleigh, Nakagami- $m, K$ and Suzuki in [19, 20]. The results of [19] show that the variance of the log-moment estimators approaches the minimal values reached by the maximum likelihood (ML) method while avoiding some of the analytical drawbacks. In contrast, as demonstrated in this paper, the method of moments (MM) applied to the NL distribution provides an unstable solution.

Motivated by the aforementioned observations, in this paper, a comprehensive analysis for the NL estimators based on the log-moments method is carried out. The advantages of the estimators are the following: (i) the parameters of the NL distribution are derived using direct equations from these estimators and therefore their calculation is significantly straightforward and simple; (ii) a unique solution of this set of parameters is found for the given estimators; and (iii) the results are robust for a range of values of NL parameters obtained across different measurement campaigns.
This paper is organized as follows: Firstly, in Section 2, the log-cumulants of the NL distribution are derived and the estimators of the NL distribution are obtained using the log-moments method. Next, in Section 3, a procedure to generate NL random variables (RVs) is derived and several numerical results are analyzed. Finally, the conclusions are discussed in Section 4.

\section{Methods}

The derivation of the log-moments of the NL distribution can be carried out by means of its log-cumulants. Therefore, firstly we obtain the log-cumulants of the NL distribution and then the estimators of the three parameters of the NL distribution are calculated by using the logmoments method. Finally, the accuracy and robustness of the fading parameter estimators of the NL distribution are briefly discussed.

\subsection{Log-cumulants of the Nakagami-lognormal Distribution}

Let $r$ be a NL RV corresponding to the received field strength expressed in $\mathrm{V} / \mathrm{m}$. The PDF of $r$ is given by ([2], (1)):

$$
\begin{aligned}
p_{r}(r)= & \int_{0}^{\infty} \frac{2}{\Gamma(m)}\left(\frac{m}{\omega}\right)^{m} r^{2 m-1} \exp \left(-\frac{m r^{2}}{\omega}\right) \\
& \times \frac{1}{\sqrt{2 \pi} \sigma \omega} \exp \left(-\frac{(\ln (\omega)-\eta)^{2}}{2 \sigma^{2}}\right) d \omega \quad r \geq 0
\end{aligned}
$$

where $m$ is the shape factor or fading parameter of the Nakagami- $m$ fast fading process, $\Gamma(z)=\int_{0}^{\infty} u^{z-1} e^{-u} d u$ is the Gamma function ([21], (6.1.1)), and:

$$
\begin{aligned}
& \eta=K \eta_{d}, \\
& \sigma=K \sigma_{d}
\end{aligned}
$$

where:

$$
K=\frac{\ln (10)}{10}
$$

$\eta_{d}$ and $\sigma_{d}$ are the mean, in $\mathrm{dBV} / \mathrm{m}$, and the standard deviation, in $\mathrm{dB}$, respectively, of the associated Gaussian shadowing process.

The cumulative distribution function (CDF) of the NL distribution can be expressed as:

$$
\begin{aligned}
F_{r}(r)= & \int_{0}^{\infty} \frac{1}{\Gamma(m)} \gamma\left(m, \frac{m}{\omega} r^{2}\right) \frac{1}{\sqrt{2 \pi} \sigma \omega} \\
& \times \exp \left(-\frac{(\ln (\omega)-\eta)^{2}}{2 \sigma^{2}}\right) d \omega \quad r \geq 0
\end{aligned}
$$

where $\gamma(a, z)=\int_{0}^{z} u^{a-1} \exp (-u) d u$ is the lower incomplete Gamma function ([21], (6.5.2)).

The $n$th moment of $r$ is calculated as: 


$$
M_{n}^{(r)}=E\left(r^{n}\right)=\frac{\Gamma\left(m+\frac{n}{2}\right)}{m^{\frac{n}{2}} \Gamma(m)} \exp \left(\frac{n}{2} \eta+\frac{n^{2}}{8} \sigma^{2}\right)
$$

where $E(\cdot)$ denotes the expectation operator. Note that (6) is equivalent to ([22], (2.59)) by substituting $2 n$ instead of $n$ in (6) which corresponds to the $n$th moment of the gamma-lognormal distribution. Thus, the first three moments of a NL distribution are given by:

$$
\begin{aligned}
& M_{1}^{(r)}=\frac{\Gamma\left(m+\frac{1}{2}\right)}{m^{\frac{1}{2}} \Gamma(m)} \exp \left(\frac{\eta}{2}+\frac{\sigma^{2}}{8}\right) \\
& M_{2}^{(r)}=\exp \left(\eta+\frac{\sigma^{2}}{2}\right) \\
& M_{3}^{(r)}=\frac{\Gamma\left(m+\frac{3}{2}\right)}{m^{\frac{3}{2}} \Gamma(m)} \exp \left(\frac{3 \eta}{2}+\frac{9 \sigma^{2}}{8}\right)
\end{aligned}
$$

In order to derive the log-moments of the NL distribution, we can calculate the first characteristic function of the second kind for the NL distribution by means of the Mellin transform framework of [19]. The first characteristic function of the second kind is defined as ([19], (8)):

$$
\phi_{r}^{(s)}=\int_{0}^{\infty} u^{s-1} p_{r}(u) d u
$$

and the first characteristic function of the second kind can alternatively be derived as ([19], (13)):

$$
\phi_{r}^{(s)}=\left.M_{n}^{(r)}\right|_{n=s-1}
$$

Substituting (6) into (11), we obtain the first characteristic function of the second kind of the NL distribution as:

$$
\phi_{r}^{(s)}=\frac{\Gamma\left(m+\frac{s-1}{2}\right)}{m^{\frac{s-1}{2}} \Gamma(m)} \exp \left(\frac{s-1}{2} \eta+\frac{(s-1)^{2}}{8} \sigma^{2}\right)
$$

The characteristic function of the second kind, defined as the natural logarithm of the first characteristic function of the second kind, can be calculated as:

$$
\Phi_{r}^{(s)}=\ln \left(\phi_{r}^{(s)}\right)
$$

The log-cumulants of order $n$ can be obtained from $\Phi_{r}^{(s)}$ as $([19],(13))$ :

$$
\kappa_{n}^{(r)}=\left.\frac{\partial^{n} \Phi_{r}^{(s)}}{\partial s^{n}}\right|_{s=1}
$$

From (12) and (13), we can calculate:

$$
\frac{\partial \Phi_{r}^{(s)}}{\partial s}=\frac{1}{2}\left(\eta+\frac{s-1}{2} \sigma^{2}-\ln (m)+\psi\left(m+\frac{s-1}{2}\right)\right)
$$

$$
\begin{aligned}
& \frac{\partial^{2} \Phi_{r}^{(s)}}{\partial s^{2}}=\frac{1}{4}\left(\sigma^{2}+\psi^{(1)}\left(m+\frac{s-1}{2}\right)\right) \\
& \frac{\partial^{n} \Phi_{r}^{(s)}}{\partial s^{n}}=\left(\frac{1}{2}\right)^{n} \psi^{(n-1)}\left(m+\frac{s-1}{2}\right), n \geq 3
\end{aligned}
$$

where $\psi(x)=\frac{\partial \ln \Gamma(x)}{\partial x}$ is the psi (digamma) function ([21], (6.3.1)) and $\psi^{(n)}=\frac{\partial^{n} \psi(x)}{\partial x^{n}}$ is the polygamma function of $n$th order ([21], (6.4.1)).

Hence, substituting $s=1$ in (15), (16) and (17), the logcumulants of the NL distribution can be derived as:

$$
\begin{aligned}
\kappa_{1}^{(r)} & =\frac{1}{2}(\eta-\ln (m)+\psi(m)) \\
\kappa_{2}^{(r)} & =\frac{1}{4}\left(\sigma^{2}+\psi^{(1)}(m)\right) \\
\kappa_{n}^{(r)} & =\left(\frac{1}{2}\right)^{n} \psi^{(n-1)}(m), n \geq 3
\end{aligned}
$$

Applying the additive properties of the log-cumulants ([19], (17)), the log-cumulant of $n$-th order in the NL distribution can also be calculated as the sum of the $\log$-cumulants of $n$-th order of both the Nakagami- $m$ and the lognormal distribution. The log-cumulants of the Nakagami- $m$ distribution are given by ([19], p. 149) and the first two log-cumulants of the lognormal distribution are given by ([23], (12)). Note that the log-cumulants of the lognormal distribution are equal to 0 for orders higher than 2 .

Using the transformation $\beta=\ln r$, the PDF of the $\log$ NL distribution can be obtained from (1) as:

$$
\begin{aligned}
p_{\beta}(\beta) & =\int_{0}^{\infty} \frac{2}{\Gamma(m)}\left(\frac{m}{\omega}\right)^{m} \exp \left(2 m \beta-\frac{m e^{2 \beta}}{\omega}\right) \\
& \times \frac{1}{\sqrt{2 \pi} \sigma \omega} \exp \left(-\frac{(\ln (\omega)-\eta)^{2}}{2 \sigma^{2}}\right) d \omega-\infty<\beta<\infty
\end{aligned}
$$

\subsection{Estimators of the Nakagami-lognormal Distribution}

The estimators of the NL distribution based on the logmoments method are derived next. In the log-moments method, the moments of the distribution in logarithmic units are related to the parameters of the distribution. Using the log-cumulants of the NL distribution derived previously, the log-moments estimators are obtained.

The relations between log-cumulants and log-moments are identical to the relations existing between moments and cumulants ([19], Section 2.3). For instance, the three first log-cumulants can be written as:

$$
\begin{aligned}
& \kappa_{1}^{(r)}=M_{1}^{(\beta)}=E(\beta) \\
& \kappa_{2}^{(r)}=\mu_{2}^{(\beta)}=E\left((\beta-\bar{\beta})^{2}\right) \\
& \kappa_{3}^{(r)}=\mu_{3}^{(\beta)}=E\left((\beta-\bar{\beta})^{3}\right)
\end{aligned}
$$

where $\beta=\ln r, M_{1}^{(\beta)}$ is the first log-moment and $\mu_{2}^{(\beta)}$ and $\mu_{3}^{(\beta)}$ are the second and third central log-moments, respectively.

In the NL distribution, from (20) and (24) with $n=3$, an estimator of $m$ can be derived by using a numerical approximation of the inverse function of $\psi^{(2)}(\cdot)$ as: 


$$
\hat{m}=1.4(-\hat{v})^{-0.391+0.0096 \ln (-\hat{v})},-16.8 \leq \hat{v}<0
$$

where $\hat{v}$ is given by:

$$
\hat{v}=8 \hat{\mu}_{3}^{(\beta)}
$$

$\hat{\mu}_{n}^{(\beta)}$ is the $n$th sample central moment of the $\log \mathrm{NL}$ distribution defined as:

$$
\begin{aligned}
\hat{\mu}_{n}^{(\beta)} & =\frac{1}{N} \sum_{i=1}^{N}\left(\beta_{i}-\hat{M}_{1}^{(\beta)}\right)^{n} \\
& =\frac{1}{N} \sum_{i=1}^{N}\left(\ln r_{i}-\hat{M}_{1}^{(\beta)}\right)^{n}
\end{aligned}
$$

where:

$$
\hat{M}_{1}^{(\beta)}=\frac{1}{N} \sum_{i=1}^{N} \ln r_{i}
$$

is the sample mean of the $\log$ NL distribution, $r_{i}, i=$ $1, \ldots, N$ are the samples of the realization corresponding to the field strength in linear units, and $N$ is the number of samples. Note that in $(25), \hat{m}(\hat{v}=-16.8) \approx 0.5$ and $\lim _{\hat{v} \rightarrow 0^{-}} \hat{m} \rightarrow \infty$.

From (18), (19), (22), and (23), we can estimate the other parameters of the NL distribution as:

$$
\begin{aligned}
& \hat{\sigma}=\sqrt{4 \hat{\mu}_{2}^{(\beta)}-\psi^{(1)}(\hat{m})} \\
& \hat{\eta}=2 \hat{M}_{1}^{(\beta)}+\ln (\hat{m})-\psi(\hat{m})
\end{aligned}
$$

Both the $\psi^{(1)}(\cdot)$ trigamma and $\psi(\cdot)$ digamma functions can be calculated using either the psi or Polygamma functions of Matlab ${ }^{\circledR}$ and Mathematica, respectively. These functions have been implemented using EulerMaclaurin summation, functional equations, and recursion ([24], p. 58).

Figure 1 shows the fading parameter estimator, $\hat{m}$, of the Nakagami- $m$ process as a function of the $\hat{v}$ estimator in logarithmic units using (25). Figure 1 is split into two plots to illustrate the small variation of $\hat{m}$ for values of $\hat{v}$ from -16.5 to -1 in the upper plot and the steep increase of $\hat{m}$ for values of $\hat{v}$ from -1 to 0 in the lower plot. The slope of this function is not substantially high for $-16.5 \leq$ $\hat{v} \leq-0.155$ which corresponds to values of $\hat{m}$ from 0.5 to 3 . For $\hat{v}$ higher than -0.155 , equivalent to $\hat{m}>3$, the slope of the function increases significantly, i.e., slight variations of $\hat{v}$ provide high modifications in the estimated values of the fading parameter. For instance, $\frac{\partial \hat{m}}{\partial \hat{v}}=22.02$ for $\hat{m}=4$.

The relative error of the expression (25) as the approximation of the inverse of $\psi^{(2)}(\cdot)$ as a function of $m$ from 0.5 to 5 is plotted in Fig. 2 using Mathematica. The maximum relative error in this interval of $m$ from 0.5 to 5 is $0.55 \%$ which occurs for $m=1.89$ and the relative error remains below $0.97 \%$ for $m$ until 23.7 .

In contrast, it can be shown that the estimators of the NL distribution using the MM (instead of the logmoments method) do not provide stable results. Specifically, in the case of the MM, from (7), (8), and (9), $\hat{m}$ can be calculated as:
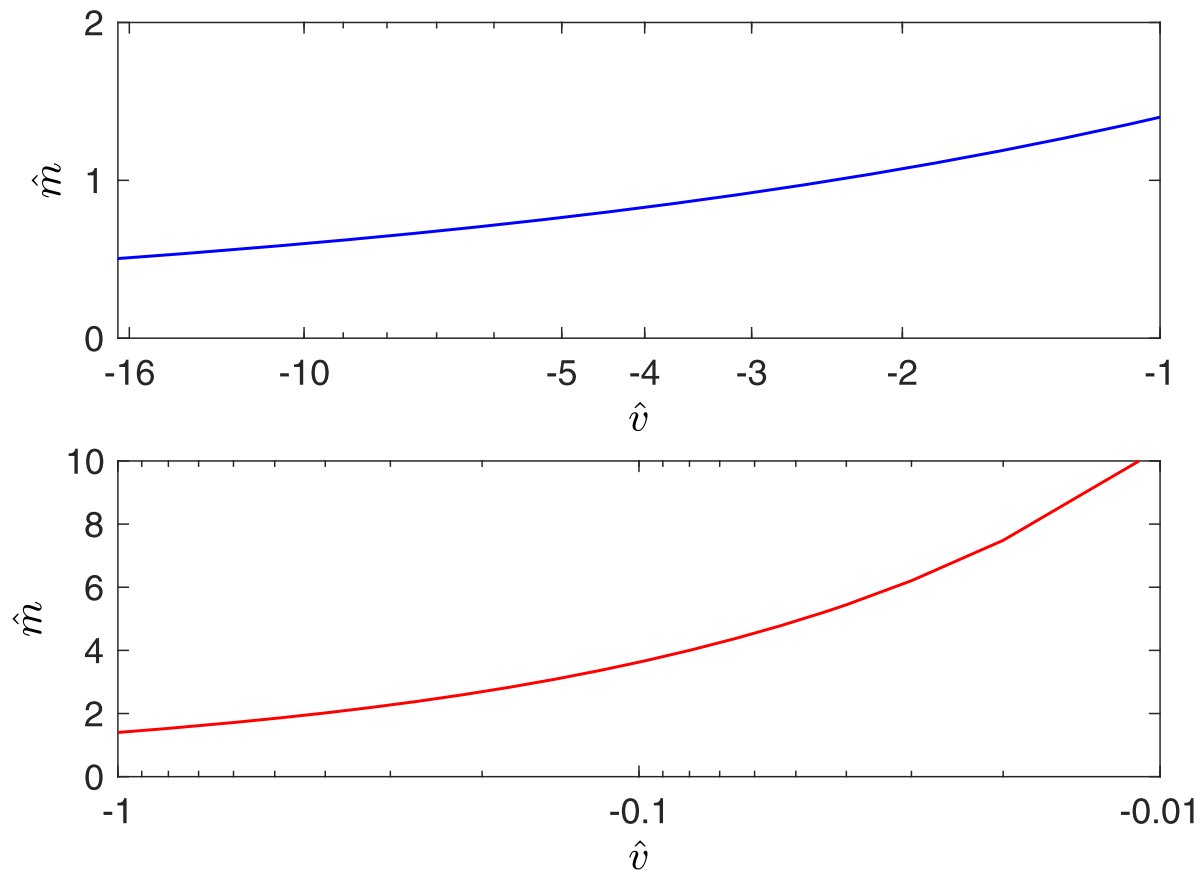

Fig. 1 Estimator of the Nakagami- $m$ fading parameter, $\hat{m}$, as a function of the $\hat{v}$ estimator 


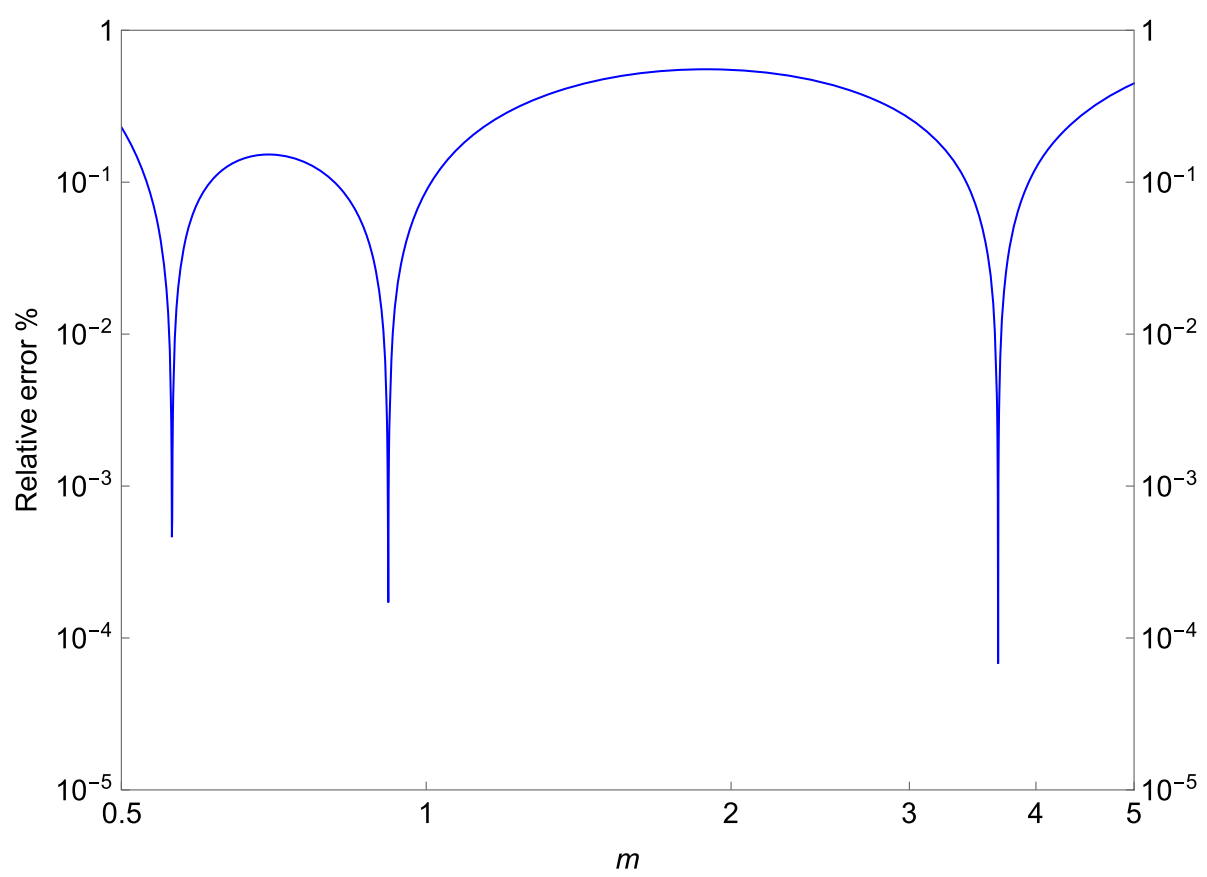

Fig. 2 Relative error of the estimator of the fading parameter, $m$, as a function of $m$

$$
\hat{m}=\frac{\exp (-1)}{\sqrt{-\hat{u}}}-0.3205^{0.0725+0.0272 \ln (-\hat{u})},-0.21 \leq \hat{u}<0
$$

where $\hat{u}$ is an estimator which uses the first three sample moments of the NL distribution in linear units as:

$$
\hat{u}=3 \ln \hat{M}_{1}^{(r)}-3 \ln \hat{M}_{2}^{(r)}+\ln \hat{M}_{3}^{(r)}
$$

where $\hat{M}_{n}^{(r)}$ is the sample $n$-th moment of the NL distribution defined as:

$$
\hat{M}_{n}^{(r)}=\frac{1}{N} \sum_{i=1}^{N} r_{i}^{n}
$$

The unreliability of $\hat{m}$ in the MM is due to the considerable slope of $\hat{m}$ as a function of $\hat{u}$. For instance, $\frac{\partial \hat{m}}{\partial \hat{u}}=46.89$ and 141.30 for $\hat{m}=2$ and $\hat{m}=3$, respectively.

On the other hand, the ML estimation method of the NL distribution leads to a set of three non-linear equations involving $N$-fold definite integrals which have to be solved by numerical methods without a unique solution. Also, numerical methods based on iterative maximization algorithms, such as those implemented in the mle function of Matlab ${ }^{\circledR}$, require starting points for the parameters and fail to converge if the initial parameter values are far from the ML estimators ([25], p. 323). This is the case for other similar lognormal-based distributions such as the three-parameter lognormal distribution where the search for the local ML estimators must be conducted with great care ([26], p. 122). Furthermore, like the MM estimators, the ML estimators of the NL distribution are also highly sensitive to slight changes of the data.

Based on these encouraging observations, we now move on to the precise evaluation of the suitability of the logmoment estimator of the NL distribution, by using the normalized mean square error (NMSE), the sample mean, and the sample confidence region introduced in the next Section.

\section{Numerical results and discussion}

In what follows, we examine the performance of the proposed estimators. This is carried out by numerically generating samples from a NL distribution. Next, the performance of the estimators will be assessed by calculating the sample mean, the sample confidence region and the NMSE for the range of parameters of the NL distribution typically found in wireless communication channels.

In order to generate $N$ NL distributed samples, first of all, $N$ Nakagami- $m$ distributed samples are generated. If $t$ is a gamma $\mathrm{RV}$, denoted by $t \sim \mathcal{G}(m, \omega)$ whose PDF is given by:

$$
p_{t}(t)=\frac{1}{\Gamma(m)}\left(\frac{m}{\omega}\right)^{m} t^{m-1} \exp \left(-\frac{m t}{\omega}\right) \quad t \geq 0
$$

then $u=\sqrt{t}$ follows a Nakagami- $m$ distribution with fading parameter $m$ and $E\left(u^{2}\right)=\omega$. Procedures of generating gamma distributed samples have been sufficiently described in the literature [27]. 
Let $u \sim \mathcal{N}(m, 1)$ be a Nakagami- $m$ RV, whose shape factor or fading parameter is $m$ and $\omega=1$. If $g$ is a Gaussian RV with mean $\eta_{d}$ and variance $\sigma_{d}^{2}$, then $r=10^{\frac{g+20 \log u}{20}}$ follows a NL RV whose PDF is given by (1).

In Fig. 3, several examples of PDFs of NL distributions are plotted in logarithmic units with $\eta_{d}=0 \mathrm{dBV} / \mathrm{m}$. The curves of the PDFs corresponding to the distributions generated following the previous procedure, depicted in dashed lines, are compared to the PDFs calculated by numerical integration of (21) plotted in solid lines. The transformation $\beta^{\prime}=(K / 2) \beta$ has been used in (21) to express the variable $\beta$ in base- 10 logarithmic units. $N=$ $100000 \mathrm{NL}$ distributed samples have been generated for different combinations of $m$ and $\sigma_{d}$, i.e., $m=3.2$ and $\sigma_{d}=6 \mathrm{~dB} ; m=1.3$ and $\sigma_{d}=8 \mathrm{~dB}$; and $m=1.3$ and $\sigma_{d}=3 \mathrm{~dB}$. From Fig. 3, the PDFs of the generated NL RVs match almost perfectly the PDFs of the analytical distribution, thus validating this process of generating samples for a NL distribution.

In order to evaluate the performance of the estimators derived in the previous section, we generate $N$ NL distributed samples and $M$ trials or realizations of each NL RV have been carried out.

Let the sample mean of a generic estimator over $M$ realizations, $\hat{b}$, be defined as:

$$
\overline{\hat{b}}_{M}=\frac{1}{M} \sum_{j=1}^{M} \hat{b}_{j}
$$

where $\hat{b}_{j}$ corresponds to the $j$ th trial of the $b$ estimator for a given distribution. The sample confidence region of the $\hat{b}$ estimator over $M$ realizations, denoted by $C_{\hat{b}_{M}}$, can be defined as:

$$
\begin{aligned}
C_{\hat{b}_{M}} & = \pm 2 \sigma_{\hat{b}_{M}}= \pm 2 \sqrt{\frac{1}{M} \sum_{j=1}^{M} \hat{b}_{j}^{2}-\overline{\hat{b}}_{M}^{2}} \\
& = \pm 2 \sqrt{\frac{1}{M} \sum_{j=1}^{M} \hat{b}_{j}^{2}-\left(\frac{1}{M} \sum_{j=1}^{M} \hat{b}_{j}\right)^{2}}
\end{aligned}
$$

where $\sigma_{\hat{b}_{M}}$ is the sample standard deviation of the $\hat{b}$ estimator over $M$ realizations. The sample confidence region is useful for examining the variations of an estimator in terms of the other estimators and the number of samples $N$ [28].

Figure 4 shows the sample mean and the sample confidence region of the estimators $\hat{m}$ and $\hat{\sigma}$ for $N=10,000$ samples and $M=10,000$ realizations. The left plot displays the sample mean and confidence region of $\hat{m}$ as derived for $\sigma_{d}=3 \mathrm{~dB}$ while the right plot displays $\hat{\sigma}$,

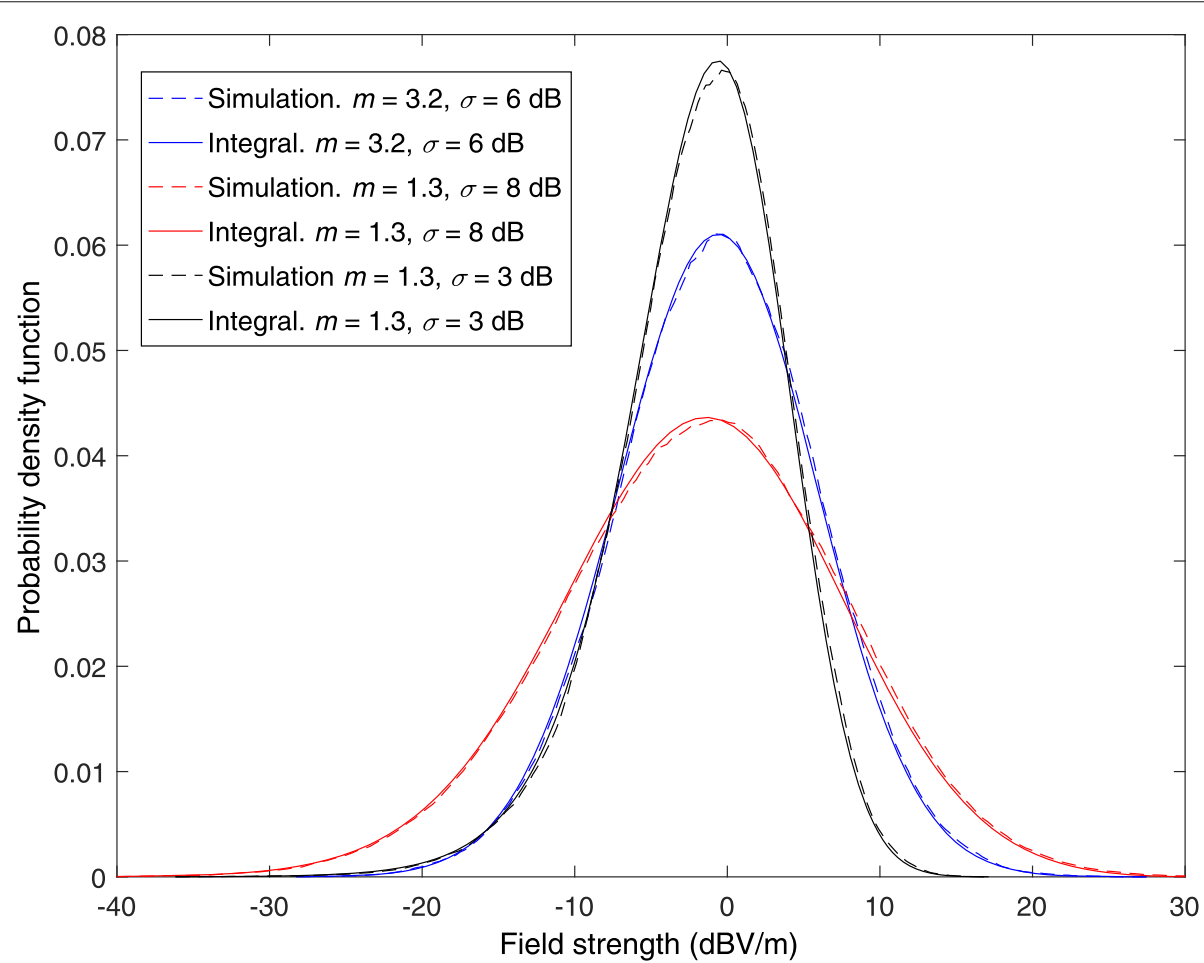

Fig. 3 Probability density functions of the Nakagami-lognormal distribution with $m=1.3, \sigma_{d}=3 \mathrm{~dB} ; m=1.3, \sigma_{d}=8 \mathrm{~dB}$; and $m=3.2, \sigma_{d}=6 \mathrm{~dB}$, respectively. Number of samples in the simulations, $N=100,000$, and $\mu_{d}=0 \mathrm{dBV} / \mathrm{m}$ 

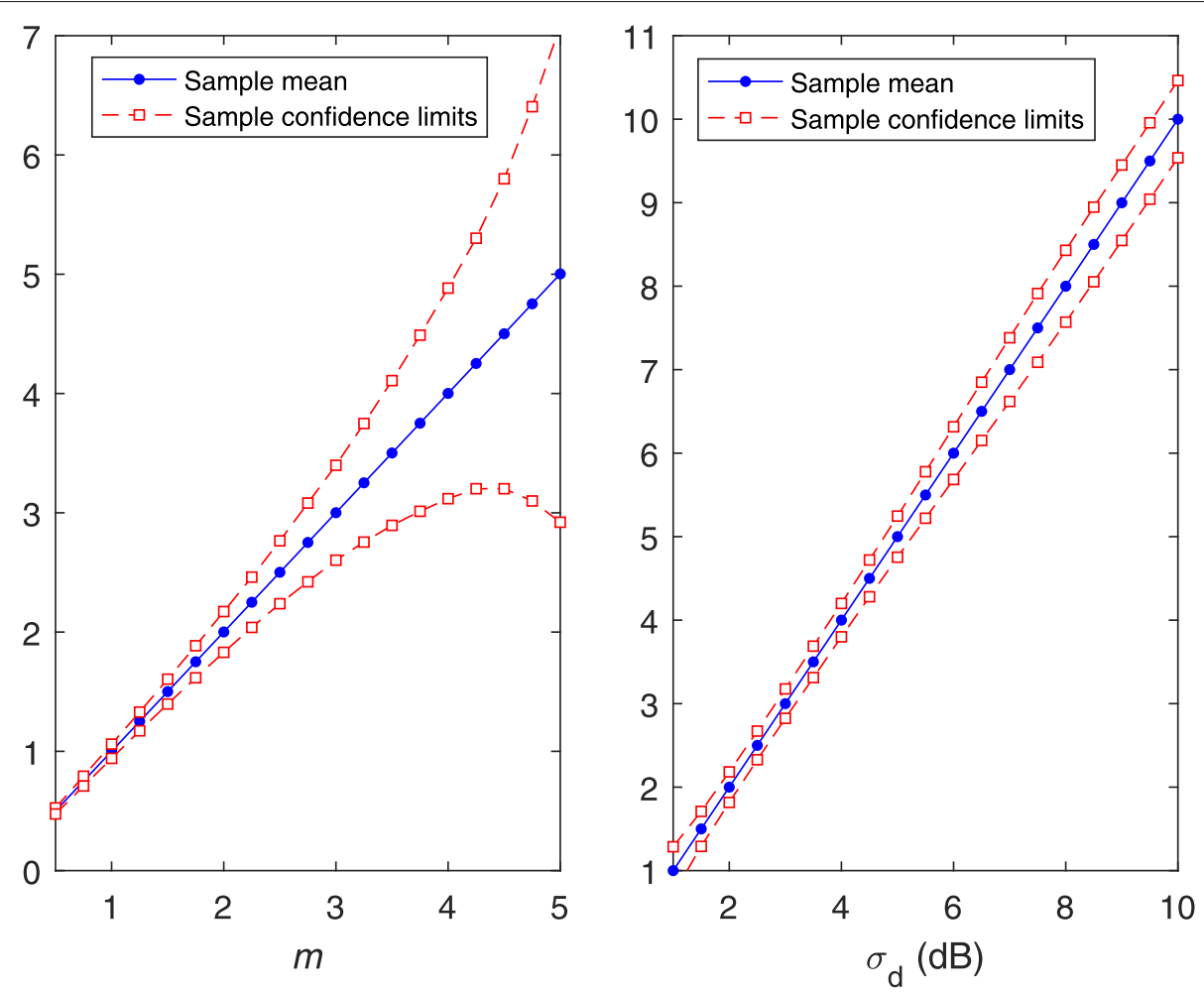

Fig. 4 Sample mean and sample confidence limits of the estimators of the fading parameter, $m$, and the standard deviation of the shadowing, $\sigma_{d}$, in the left plot and the right plot, respectively. Number of samples $=10,000$ and number of trials $M=10,000$. In the left plot, $\sigma_{d}=3 \mathrm{~dB}$. In the right plot, $m=2.3$

as derived for an assumed value of $m=2.3$. It can be seen that both estimators are not biased since the slope of the sample means is equal to 1 . From the left plot, it can be inferred that the confidence region is drastically increased for fading parameters, $m$, higher than 4.3. Nevertheless, from values reported in a measurement campaign conducted in a macrocellular environment, the fading parameter typically ranges from 0.5 to 3.5 [29], and therefore, we expect this estimator to be accurate when estimating the fading parameter from real wireless channel measurements. In the right plot, it can be shown that the confidence region does not vary substantially in the range from 0.5 to $10 \mathrm{~dB}$ with only a slight increase.

The NMSE has been traditionally used to measure the quality of an estimator [25]. In the case of an unbiased estimator, the NMSE corresponds to the normalized sample variance. We can define the NMSE of a generic estimator, $\hat{b}$, over $M$ realizations as:

$$
\operatorname{NMSE}_{M}(\hat{b})=\frac{1}{M b^{2}} \sum_{j=1}^{M}\left(\hat{b}_{j}-b\right)^{2}
$$

where $b$ is the parameter of the distribution generated and $\hat{b}_{j}, j=1, \ldots, M$ is the estimated parameter in the $j$ th trial.

In order to calculate the NL estimators with a sufficient accuracy, a minimum number of samples should be taken from a measurement record. In what follows, a threshold of NMSE equal to $10^{-1}$ has been used.

Figure 5 shows the NMSE of the $\hat{m}$ estimator as a function of the number of samples, $N$, from $N=1000$ to 100,000 , values of the fading parameter $m=0.5,2.3$, and 3.5, and standard deviations of the associated Gaussian process $\sigma_{d}=3$ and $6 \mathrm{~dB}$. Curves have been plotted for $M=30,000$ trials. As expected, the NMSE of the $\hat{m}$ estimator decreases with the number of samples, except for $m=3.5$ and number of samples $N \leq 8000$. In order to achieve NMSE $\leq 10^{-1}$, if $m=3.5$, the number of samples needs to be higher than 4000 and 76,000 for $\sigma_{d}=3$ and $6 \mathrm{~dB}$, respectively. In the case of $m=$ 2.3 , the number of samples for NMSE $\leq 10^{-1}$ needs to be larger than 1500 and 16,000 for $\sigma_{d}=3$ and 6 $\mathrm{dB}$, respectively. In order words, the accuracy of the $\hat{m}$ estimator is significantly high for small fading parameters and even a low number of samples of the measurement record. However, the number of samples required for the threshold NMSE of $10^{-1}$ can be medium-high if both the fading parameter and the standard deviation of the shadowing are large. Furthermore, generally speaking, the NMSE of the $\hat{m}$ estimator increases as the standard deviation of the lognormal distribution grows, except for $m=3.5$ and $N \leq 1190$ samples in this case. 


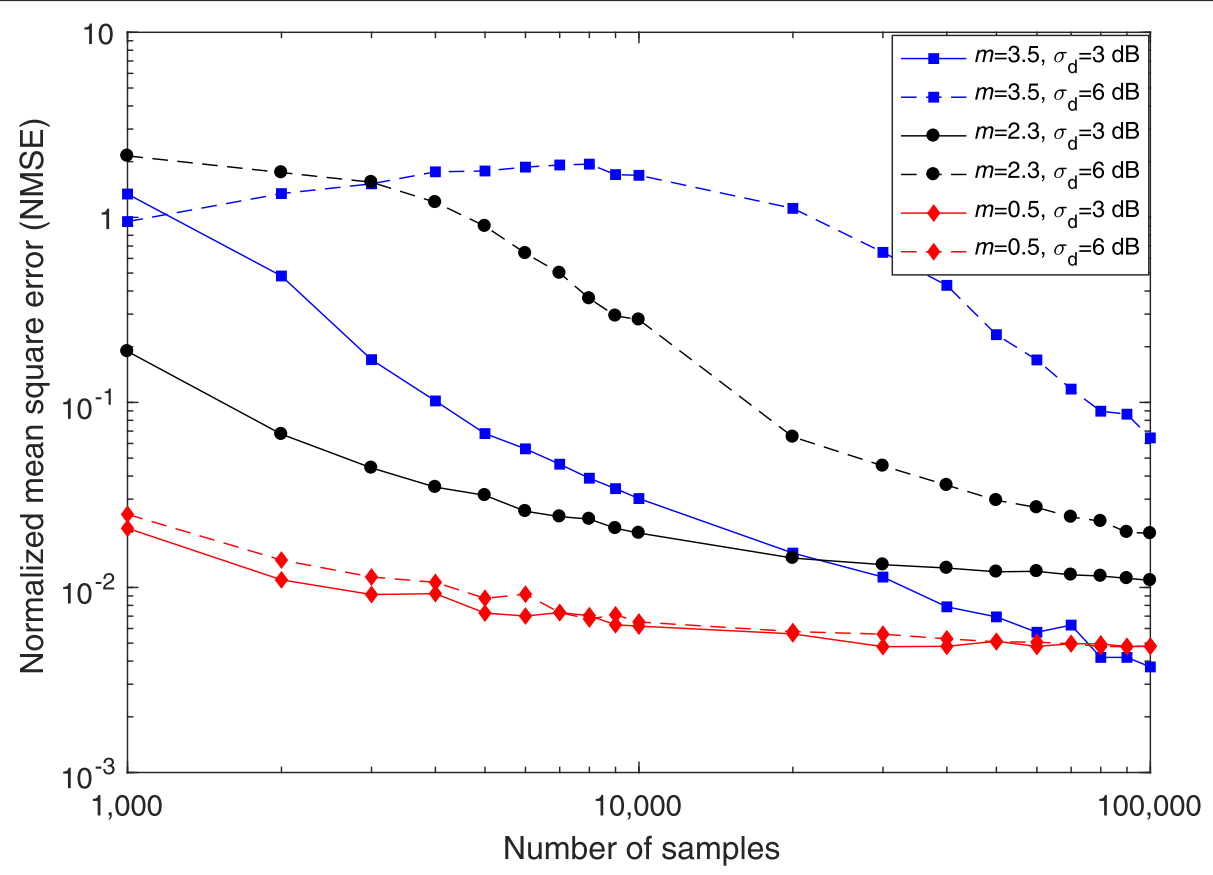

Fig. 5 Normalized mean square error of the $\hat{m}$ estimator of the fading parameter for the Nakagami- $m$ process, as a function of the number of samples, $N$, for $m=0.5,2.3$, and 3.5 and standard deviations of the Gaussian process, $\sigma_{d}=3 \mathrm{~dB}$ and $\sigma_{d}=6 \mathrm{~dB}$

Also, the NMSE of the $\hat{m}$ increases substantially with the fading parameter $m$ for a sufficient number of samples. Nevertheless, the slope of the NMSE of $\hat{m}$ as a function of the number of samples in logarithmic units tends to stabilize for a number of samples given which increases with $\hat{m}$, independently of the standard deviation of the shadowing, $\sigma_{d}$.

In Fig. 6, the NMSE of the standard deviation estimator, $\hat{\sigma}_{d}$, is plotted as a function of the standard deviation in $\mathrm{dB}, \sigma_{d}$, for $N=10,000$ samples and $M=30,000$ trials.

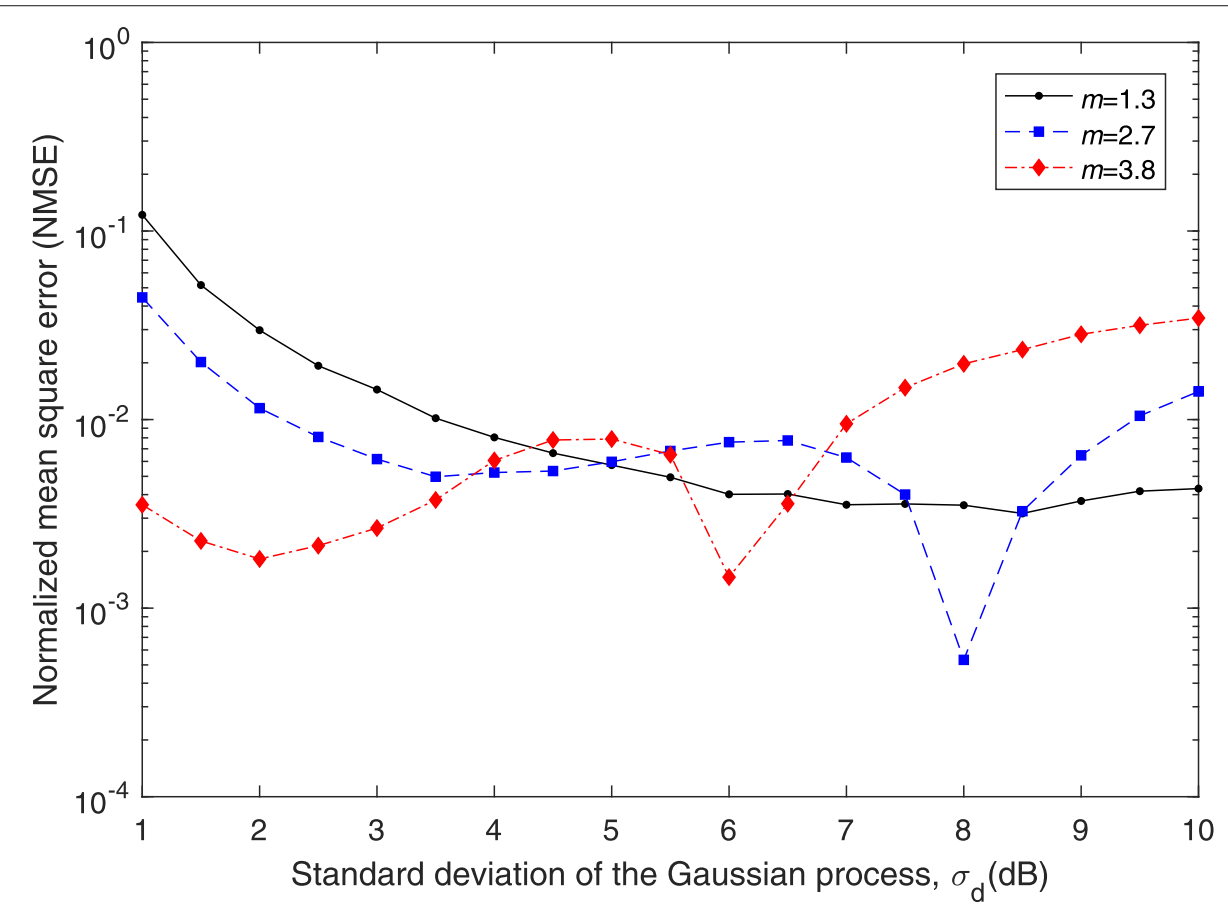

Fig. 6 Normalized mean square error of the $\hat{\sigma}_{d}$ estimator as a function of the standard deviation of the Gaussian process for $N=10,000$ samples and fading parameters of the Nakagami- $m$ process, $m=1.3,2.7$ and 3.8 
The values of the fading parameter of the Nakagami$m$ process used in the simulations are $m=1.3,2.7$, and 3.8. From these curves, it can be shown that the NMSE remains below $10^{-1}$, except for $\sigma_{d} \leq 1.1 \mathrm{~dB}$ and $m=1.3$ even though the number of samples is not excessively high, i.e., $N=10,000$, compared to that required for the same value of the NMSE in the $\hat{m}$ estimator.

\section{Conclusions}

In this paper, we have derived log-moment estimators for the NL distribution. Moreover, the performance of those estimators has been extensively analyzed. The results show that the NMSE of the fading parameter estimator is very dependent on both the shadowing standard deviation and the fading parameter even when the number of samples is significant, $N=100,000$. This NMSE increases substantially as either the shadowing standard deviation or the fading parameter of the Nakagami- $m$ distribution grow, with values of NMSE of the fading parameter estimator higher than $10^{-1}$ for fading parameters larger than approximately 2 , a shadowing standard deviation of $6 \mathrm{~dB}$ and $N=10,000$ number of samples. On the other hand, the NMSE of the shadowing standard deviation estimator remains significantly small for a number of samples not excessively high, i.e., $N=10,000$, with values ranging from $5.3 \times 10^{-4}$ to $1.2 \times 10^{-1}$ for $m=2.7, \sigma_{d}=8 \mathrm{~dB}$ and $m=1.3, \sigma_{d}=1 \mathrm{~dB}$, respectively.

\section{Abbreviations \\ AFD: Average time fade duration; ASEP: Average symbol error probability; ASM: Adaptive selection/maximal-ratio; BER: Bit error rate; CDF: Cumulative distribution function; DPSK: Differential phase-shift keying; LCR: Level crossing rate; MGF: Moment generating function; ML: Maximum likelihood; MM: Moments method; MPSK: M-ary phase shift keying; MRC: Maximal ratio combining; NCFSK: Non-coherent frequency-shift keying; NL: Nakagami-lognormal; NMSE: Normalized mean square error; PDF: Probability density function; RV: random variable; SNR: signal-to-noise ratio}

\section{Acknowledgments}

Not applicable.

\section{Funding}

This work has been funded in part by the Programa de Estancias de Movilidad de Profesores e Investigadores en Centros Extranjeros de Enseñanza Superior e Investigación of the Ministerio de Educación, Cultura y Deporte, Spain, PR2015-00151 and by the Ministerio de Economía, Industria y Competitividad of the Spanish Government under the national project TEC2017-86779-C2-2-R, through the Agencia Estatal de Investigación (AEI) and the Fondo Europeo de Desarrollo Regional (FEDER).

\section{Availability of data and materials}

Data sharing is not applicable to this article as no datasets were generated or analyzed during the current study.

\section{Authors' contributions}

$J R$ performed the mathematical derivation of the Nakagami-lognormal estimators and was a major contributor in writing the manuscript. CB, VMR, and $L R$ carried out the simulations of the numerical results. All authors interpreted the results and read and approved the final manuscript.

\section{Authors' information}

Juan Reig received his Ph.D. from the Universitat Politècnica de València (UPV) Spain, in 2000. He has been a faculty member in the Department of Communications at UPV since 1994, where he is now Professor of Telecommunication Engineering. He is a member of the Electromagnetic Radiation Group (GRE) of the Institute of Telecommunications and Multimedia Applications (iTEAM). His areas of interest include fading theory, diversity, ultrawide band (UWB) systems, vehicular communications, and millimeter wave (mmWave) propagation. Conor Brennan received a B.A. Mod (Hons) and gold medal in mathematics from Trinity College Dublin (TCD) in 1994 and a PhD in 1998 from the same institution. He joined Dublin City University as an Assistant Professor in 2003 and became an Associate Professor in 2013. Brennan serves on the Royal Irish Academy Committee on Engineering and Computer Science and was General Chair of its 2017 URSI Radio Science research colloquium "Radio Science and Communications for a Smarter World". His primary research area is in computational methods for electromagnetic wave propagation and scattering, and applying such research to associated problems such as indoor user location and tracking, energy efficient wireless communications and medical imaging. Vicent Miquel Rodrigo Peñarrocha received his PhD in 2003 from the UPV. He joined the Department of Communications at the UPV in 1991 as a Lecturer. His current interests include radiowave propagation, antenna measurements, instrumentation, virtual instrumentation and laboratories and any educational activity. Lorenzo Rubio received his Ph.D degree from the UPV, Spain, in 2004. In 1996, he joined the Department of Communications at UPV, where he is now Associate Professor of wireless communications. He is a member of the GRE of the iTEAM. His main research interests are related to wireless communications. Specific current research topics include radiowave propagation, measurement and mobile time-varying channels modeling in vehicular applications, UWB communication systems, multiple-input and multiple-output (MIMO) systems, equalization techniques in digital wireless systems and mmWave propagation.

\section{Competing interests}

The authors declare that they have no competing interests.

\section{Publisher's Note}

Springer Nature remains neutral with regard to jurisdictional claims in published maps and institutional affiliations.

\section{Author details}

${ }^{1}$ Electromagnetic Radiation Group (GRE), Institute of Telecommunications and Multimedia Applications (iTEAM), Universitat Politècnica de València, Camino de Vera, 46022 Valencia, Spain. ${ }^{2}$ School of Electronic Engineering, Dublin City University, Glasnevin, Dublin 9, Ireland.

Received: 18 July 2018 Accepted: 14 December 2018

Published online: 09 January 2019

References

1. J. M. Ho, G. L. Stüber, in Co-channel interference of microcellular systems on shadowed Nakagami fading channels. Proc. IEEE 43rd Vehicular Technology Conference, 1993 (VTC 93) (IEEE, Secaucus, 1993), pp. 568-571

2. A. A. Abu-Dayya, N. C. Beaulieu, Micro- and macrodiversity NCFSK (DPSK) on shadowed Nakagami-fading channels. IEEE Trans. Commun. 42(9), 2693-2702 (1994)

3. X. Wang, W. Wang, Z. Bu, Fade statistics for selection diversity in Nakagami-lognormal fading channels. Electron. Lett. 42(18), 1046-1047 (2006)

4. D. T. Nguyen, Q. T. Nguyen, S. C. Lam, Analysis and simulation of MRC diversity reception in correlated composite Nakagami-lognormal fading channels. REV J. Electron. Commun. 4(1-2), 44-51 (2014)

5. P. Xu, X. Zhou, D. Hu, in Performance evaluations of adaptive modulation over composite Nakagami-lognormal fading channels. 2009 15th Asia-Pacific Conference on Communications (IEEE, Shanghai, 2009), pp. $467-470$

6. G. C. Alexandropoulos, A. Conti, P. T. Mathiopoulos, in Adaptive M-QAM systems with diversity in correlated Nakagami-m fading and shadowing. IEEE Global Telecommunications Conference (GLOBECOM 2010) (IEEE, Miami, 2010), pp. 1-5 
7. Ö. Bulakci, A. B. Saleh, J. Hämäläinen, S. Redana, Performance analysis of relay site planning over composite fading/shadowing channels with cochannel interference. IEEE Trans. Veh. Technol. 62(4), 1692-1706 (2013)

8. W. Cheng, Y. Huang, On the performance of adaptive SC/MRC cooperative systems over composite fading channels. Chin. J. Electron. 25(3), 533-540 (2016)

9. M. G. Kibria, G. P. Villardi, W. Liao, K. Nguyen, K. Ishizu, F. Kojima, Outage analysis of offloading in heterogeneous networks: Composite fading channels. IEEE Trans. Veh. Technol. 66(10), 8990-9004 (2017)

10. K. Cho, J. Lee, C. G. Kang, Stochastic geometry-based coverage and rate analysis under Nakagami \& log-normal composite fading channel for downlink cellular networks. IEEE Commun. Lett. 21(6), 1437-1440 (2017)

11. R. Singh, M. Rawat, Closed-form distribution and analysis of a combined Nakagami-lognormal shadowing and unshadowing fading channel. J Telecommun. Inf. Technol. 4, 81-87 (2016)

12. J. Reig, L. Rubio, Estimation of the composite fast fading and shadowing distribution using the log-moments in wireless communications. IEEE Trans. Wireless. Commun. 12(8), 3672-3681 (2013)

13. S. Atapattu, C. Tellambura, H. Jiang, A mixture gamma distribution to model the SNR of wireless channels. IEEE Trans. Wireless Commun. 10(12), 4193-4203 (2011)

14. Q. Wang, H. Lin, P. Kam, Tight bounds and invertible average error probability expressions over composite fading channels. J. Commun. Netw. 18(2), 182-189 (2016)

15. J. M. Holtzmann, On using perturbation analysis to do sensitivity analysis: derivatives versus differences. IEEE Trans. Autom. Control. 37(2), 243-247 (1992)

16. H. Suzuki, A statistical model for urban radio propagation. IEEE Trans. Commun. 25(7), 673-680 (1977)

17. M. D. Yacoub, The $\alpha-\mu$ distribution: a physical fading model for the Stacy distribution. IEEE Trans. Veh. Technol. 56(1), 122-124 (2007)

18. P. M. Shankar, Error rates in generalized shadowed fading channels. Wirel. Pers. Commun. 28(3), 233-238 (2004)

19. J.-M. Nicolas, Introduction aux statistiques de deuxième espèce: applications des logs-moments et des logs-cumulants à l'analyse des lois d'images radar. Traitement du Signal. 19(3), 139-167 (2002). Translation to English by S. N. Anfinsen

20. C. Withers, S. Nadarajah, A generalized Suzuki distribution. Wirel. Pers. Commun. 62(4), 807-830 (2012)

21. M. Abramowitz, Handbook of Mathematical Functions, with Formulas, Graphs, and Mathematical Tables, 9th edn. (Dover, New York, NY, 1972)

22. M. K. Simon, M. S. Alouini, Digital Communication over Fading Channels, 2nd edn. (Wiley, Hoboken, NY, 2005)

23. Z. Sun, J. Du, in Proc. 10th International Conference, ICIC 2014, ed. by D.-S. Huang, V. Bevilacqua, and P. Premaratne. Log-cumulant parameter estimator of log-normal distribution. Intelligent computing theory (Springer, New York, NY, 2014), pp. 668-674

24. S. Zhang, J. M. Jin, Computation of Special Functions. (Wiley, New York, 1996)

25. G. Casella, R. L. Berger, Statistical Inference. (Duxbury Thomson Learning, Pacific Grove, CA, 2002)

26. C. Kleiber, S. Kotz, Statistical Size Distributions in Economics and Actuarial Sciences. (Wiley, Hoboken, NJ, 2003)

27. L. Devroye, Non-uniform Random Variate Generation. (Springer, New York,1986)

28. A. Abdi, M. Kaveh, Performance comparison of three different estimators for the Nakagami $m$ parameter using Monte Carlo simulation. IEEE Commun. Lett. 4(4), 119-121 (2000)

29. L. Rubio, J. Reig, N. Cardona, Evaluation of Nakagami fading behaviour based on measurements in urban scenarios. Int. J. Electron. Commun. (AEÜ). 61(2), 135-138 (2007)

\section{Submit your manuscript to a SpringerOpen ${ }^{\circ}$ journal and benefit from:}

- Convenient online submission

- Rigorous peer review

- Open access: articles freely available online

- High visibility within the field

- Retaining the copyright to your article

Submit your next manuscript at $>$ springeropen.com 